\title{
Energy loss, a novel biomechanical parameter, correlates with aortic aneurysm size and histopathologic findings
}

\author{
Jennifer Chung, MD, ${ }^{\mathrm{a}}$ Kevin Lachapelle, MD, ${ }^{\mathrm{a}}$ Evan Wener, BASc, ${ }^{\mathrm{c}}$ Raymond Cartier, MD, ${ }^{\mathrm{d}}$ \\ Benoit De Varennes, MD, ${ }^{\mathrm{a}}$ Richard Fraser, MD, MSc, ${ }^{\mathrm{b}}$ and Richard L. Leask, $\mathrm{PhD}^{\mathrm{c}, \mathrm{d}}$
}

\begin{abstract}
Objective: Energy loss is a biomechanical parameter that represents the relative amount of energy absorbed by the aorta during the cardiac cycle. We aimed to correlate energy loss with ascending aortic aneurysm size and histopathologic findings to elucidate the pathophysiology of aneurysm complications.
\end{abstract}

\begin{abstract}
Methods: Aneurysmal ascending aortic specimens were obtained during surgery. Control specimens were obtained from autopsy and organ donors. Biaxial tensile tests were performed on the 4 quadrants of the aortic ring. Energy loss was calculated using the integral of the stress-strain curve during loading and unloading. It was compared with the size and the traditional biomechanical parameter, stiffness (apparent modulus of elasticity). Elastin, collagen, and mucopolysaccharide content were quantified using Movat pentachrome staining of histology slides.
\end{abstract}

Results: A total of 41 aortas were collected ( 34 aneurysmal, 7 control). The aneurysms exhibited increased stiffness $(P<.0001)$ and energy loss $(P<.0001)$ compared with the controls. Energy loss correlated significantly with aortic size $\left(P<.0001, r^{2}=.60\right)$. A hinge point was noted at a diameter of $5.5 \mathrm{~cm}$, after which energy loss increased rapidly. The relationship between energy loss and size became strongly linear once the size was indexed to the body surface area $\left(P<.0001, r^{2}=.78\right)$. Energy loss correlated with the histopathologic findings, especially the collagen/elastin ratio $\left(P=.0002, r^{2}=.49\right)$. High energy loss distinguished patients with pathologic histologic findings from others with similar diameters.

Conclusions: As ascending aortas dilate, they exhibit greater energy loss that rapidly increases after $5.5 \mathrm{~cm}$. This mirrors the increase in complications at this size. Energy loss correlates with imbalances in elastin and collagen composition, suggesting a measurable link between the histopathologic features and mechanical function. (J Thorac Cardiovasc Surg 2014;148:1082-9)

The catastrophic complications associated with aneurysms of the ascending aorta are preventable with elective surgery. However, identifying patients for surgery can be challenging. The present guidelines predominantly used size alone and low levels of evidence. ${ }^{1}$ A recent study found wide variations across Canada in what surgeons believe to be the appropriate size criteria for bicuspid aortopathy. ${ }^{2}$ Given the mechanical endpoints of aortic dissection and rupture,

From the Divisions of Cardiac Surgery ${ }^{\mathrm{a}}$ and Pathology, ${ }^{\mathrm{b}}$ and Department of Chemical Engineering, ${ }^{\mathrm{c}}$ McGill University, Montreal, Quebec, Canada; and Montreal Heart Institute, ${ }^{\mathrm{d}}$ Montreal, Quebec, Canada.

This study was supported by the Heart and Stroke Foundation of Quebec (\#235502 and \#236951).

Disclosures: Kevin Lachapelle reports consulting fees from Medtronic, Covidien, and the Royal College. Benoit De Varennes reports lecture fees and grant support from Medtronic. All other authors have nothing to disclose with regard to commercial support.

Read at the 94th Annual Meeting of The American Association for Thoracic Surgery, Toronto, Ontario Canada, April 26-30, 2014.

Received for publication April 16, 2014; revisions received May 29, 2014; accepted for publication June 10, 2014.

Address for reprints: Richard L. Leask, PhD, Department of Chemical Engineering, McGill University, Wong Bldg, Rm 4120, 3610 rue University Montreal Quebec Canada, Montreal, Quebec H3A 0C5, Canada (E-mail: richard.leask@mcgill.ca). 0022-5223/\$36.00

Crown Copyright $\odot 2014$ Published by Elsevier Inc. on behalf of The American Association for Thoracic Surgery

http://dx.doi.org/10.1016/j.jtcvs.2014.06.021 measuring and understanding the biomechanical changes associated with aneurysms is fundamental to identifying at-risk aortas. Biomechanical metrics could provide a more precise prediction model and optimize surgical selection.

Currently, size is used as a surrogate biomechanical metric. However, small aortas can experience complications, and large aortas can remain stable. The International Registry of Acute Aortic Dissection database revealed aortic size to be a poor predictor of dissection. ${ }^{3}$ Size also fails to describe the quality of the aortic wall. Thus, many have quantified aortic stiffness using the apparent modulus of elasticity extracted from ex vivo mechanical testing or estimated from in vivo imaging. ${ }^{4-6}$ Aneurysmal tissue is stiffer than normal aortic tissue., ${ }^{4,7}$ However, increased stiffness alone fails to explain why aneurysms have a propensity for complications rather than greater resilience. Moreover, the nonlinear material properties of the aorta make the apparent modulus dependent on the level and rate of strain experienced by the aorta. The ultimate tensile strength usually exceeds physiologic limits and is only measureable ex vivo. ${ }^{8,9}$

We examined the biomechanical parameter energy loss, defined as hysteresis normalized to total stored strain energy, and its relationship to aortic size and histopathologic features. This parameter draws from the Windkessel function 
of the ascending aorta, which must expand and act as a capacitor during systole and recoil and return the stored energy to the circulation during diastole. Greater energy loss represents greater inefficiency in performing this function and greater energy dissipated into the aortic wall. This could lead to eventual aortic remodeling and failure. Unlike the discrete nature of the apparent modulus of elasticity, which uses an arbitrary point for measurement, the energy loss parameter integrates the stress-strain relationship of the tissue over the entire cycle, making it a more stable metric.

In a first step toward validating a clinically measurable biomechanical risk factor for aneurysmal aortas, we measured the energy loss and apparent modulus of ascending aortic tissue ex vivo. We hypothesized that energy loss would be linked to aortic size, that the structural compositional changes in the aneurysmal aortic walls would be associated with increased energy loss, and that energy loss can be used to identify aortas with pathologic histologic changes.

\section{METHODS}

Excised aneurysmal ascending aortic tissue was obtained at surgery from July 2012 to May 2013 at McGill University Health Centre and Montreal Heart Institute. Control ascending aortic tissue was obtained from heart transplant donors and autopsy patients without heart or aortic disease. The research ethics board at both hospitals approved the study.

\section{Biaxial Tensile Testing}

Testing was done within 24 hours of tissue collection, and the specimens were kept on ice until testing was completed. All samples were collected as intact rings, with orientation marked by a single clip. Four $1.5-\mathrm{cm}$ by $1.5-\mathrm{cm}$ squares were collected along the belly of the aneurysm. Each square underwent uni- and equibiaxial tensile testing at $37^{\circ} \mathrm{C}$ in a Ringer's lactate solution to a maximum of $60 \%$ strain (EnduraTEC ELF 3200; Bose Corp, Framingham, Mass). We have previously shown that in healthy pig ascending aortic tissue, smooth muscle cell activation with phenylephrine does affect the stiffness of the tissue; however, the contribution is limited at high strains to $<10 \%$ of the stiffness increase. ${ }^{10}$ For consistency, smooth muscle cells were not activated in our experiments. Ten preconditioning loops were completed before the test loops, which were done in triplicate. Both engineering and true stress using optical tracking were measured.

Analysis was performed using MatLab R2012a (MathWorks, Natick, Mass). Both the engineering stress and true-stress-strain curves were modeled using polynomial functions. The apparent modulus of elasticity was calculated at $40 \%$ and $50 \%$ strain as an approximation of physiologic loading conditions. We measured the percentage of strain energy lost between the loading and unloading curves, "energy loss," to quantify the viscoelastic nature of the aorta (Figure 1). Loading and unloading of aortic tissue produces a hysteresis loop in the stress-strain curve. The area of this loop has units of energy. Once hysteresis has been normalized by dividing by the total energy applied during loading (the integral of the loading stress-strain curve), this will yield a unit-less metric termed "energy loss." The data and figures presented consistently used strain in the axial direction during equibiaxial testing and engineering stress.

\section{Histologic Assessment}

Samples immediately adjacent to each square were stored in $10 \%$ formalin and underwent bulk Movat Pentachrome staining. Images were taken in 3 different areas of each slide, for a total of 12 images per aorta. All images were renamed and analyzed in a blinded fashion. The

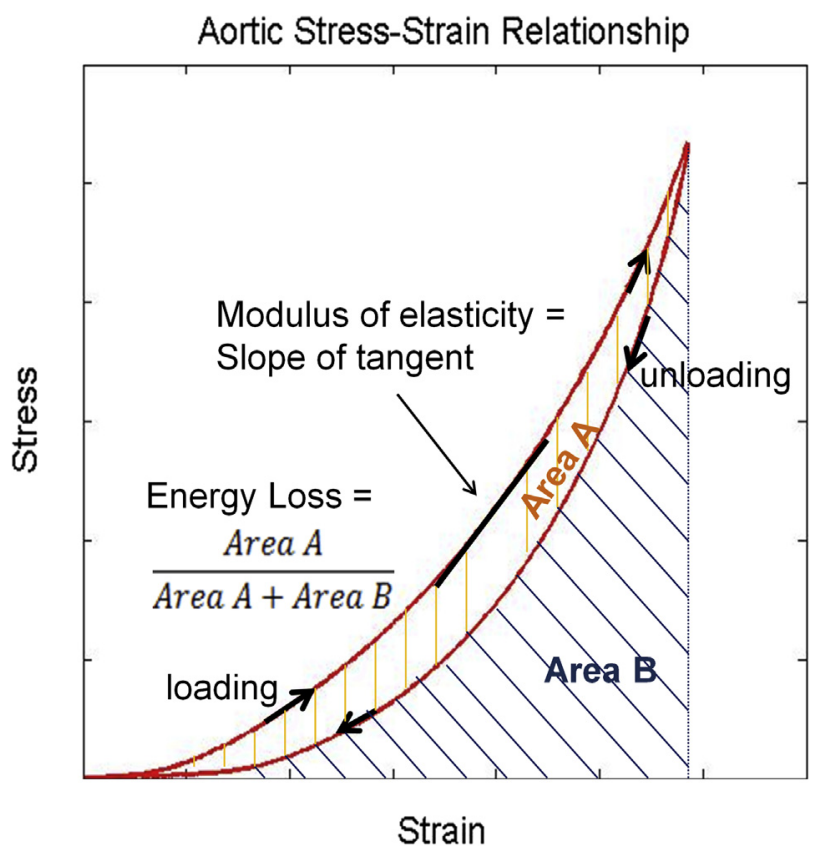

FIGURE 1. The stress-strain relationship of the ascending aorta and definitions of 2 biomechanical parameters: the apparent modulus of elasticity and the percentage of energy loss per cycle.

percentage of collagen, elastin, and mucopolysaccharide were quantified using ImageJ 1.46r (National Institutes of Health, Bethesda, Md).

\section{Statistical Analysis}

All averages are presented as the mean \pm standard deviation. Continuous variables were compared using the Mann-Whitney $U$ test. Correlations were calculated using linear regression. The body surface area was calculated using the Dubois formula:

$$
B S A=0.20247 \times H^{0.725} \times W^{0.425}
$$

where BSA is the body surface area in square meters, $\mathrm{H}$ is the height in meters, and $\mathrm{W}$ is the weight in kilograms.

Statistical analyses were performed using GraphPad Prism, version 5.01 (GraphPad Software, Inc, San Diego, Calif).

\section{RESULTS}

A total of 41 ascending aortas were collected, including 34 aneurysmal aortas and 7 control aortas (Table 1). We found statistically significant differences in the biomechanical properties between the aneurysmal and control aortas. The aneurysmal tissue, with an mean energy

TABLE 1. Patient demographics

\begin{tabular}{lcc}
\hline \multicolumn{1}{c}{ Variable } & Control $(\mathbf{n}=\mathbf{7})$ & Aneurysm $(\mathbf{n}=\mathbf{3 4})$ \\
\hline Age $(\mathrm{y})$ & $51 \pm 18$ & $64 \pm 12$ \\
Male sex & $6(86)$ & $23(68)$ \\
Tricuspid aortic valves & $7(100)$ & $12(35)$ \\
Bicuspid aortic valves & 0 & $20(59)$ \\
Mechanical valves & 0 & $2(6)$ \\
Aortic size $(\mathrm{cm})$ & $2.4 \pm 0.4$ & $5.2 \pm 0.7$ \\
\hline
\end{tabular}



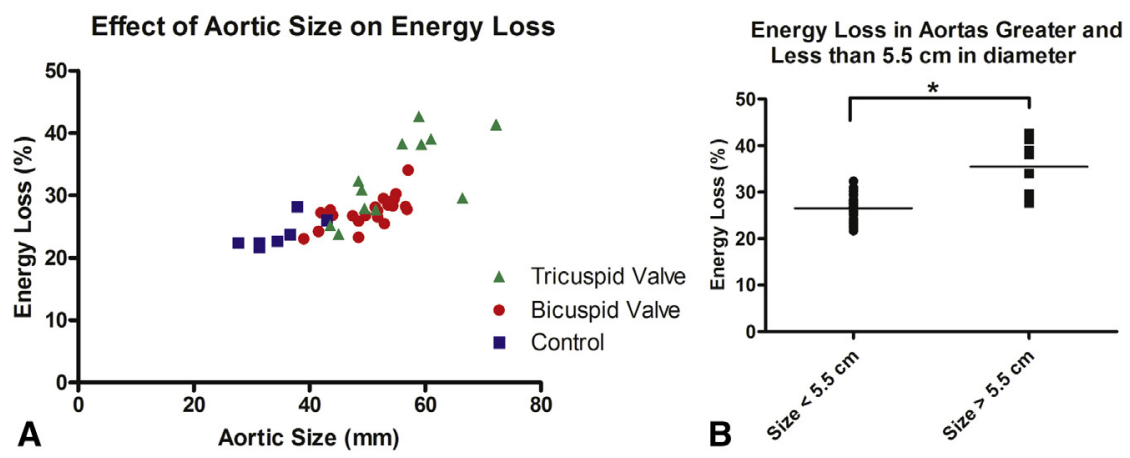

FIGURE 2. A, The effect of aortic diameter on energy loss. B, Energy loss rapidly increases with greater variability for aortic diameters $>55 \mathrm{~mm}$. The average energy loss in aortas $<55 \mathrm{~mm}$ and $>55 \mathrm{~mm}$ was $26.5 \% \pm 2.7 \%$ and $35.5 \% \pm 5.8 \%$, respectively $(P=.0001)$.

loss of $34 \% \pm 4 \%$, exhibited significantly greater energy loss than control tissue, with a mean of $28 \% \pm 2 \%$ $(P=.0005)$. The aneurysmal tissue also exhibited a significantly greater apparent modulus of elasticity at $40 \%$ strain compared with the control tissue (aneurysmal, $0.15 \pm 0.03 \mathrm{MPa}$; control, $0.10 \pm 0.03 \mathrm{MPa} ; P=.0007$ ). When separating the aneurysmal aorta between the tricuspid and bicuspid valves, no difference was found in the apparent modulus of elasticity between the 2 valve types $(P=.77)$, although the bicuspid valves exhibited less energy loss than the tricuspid valves $(P=.01)$.

We explored the relationship between the biomechanical parameters and aortic size. A trend was seen toward a greater apparent modulus of elasticity, or aortic stiffness, with increasing aortic size $(40 \%$ strain, $P=.001$, $r^{2}=.17 ; 50 \%$ strain, $P=.0002, r^{2}=.30$ ). However, the trend disappeared once the aortic size was indexed to the body surface area $\left(40 \%\right.$ strain, $P=.82, r^{2}=.002 ; 50 \%$ strain, $\left.P=.07, r^{2}=.11\right)$. Energy loss and aortic size significantly co-varied, with greater energy loss seen in larger aortas $\left(P<.0001, r^{2}=.60\right)$. When the aortic size increased to $>5.5 \mathrm{~cm}$, the energy loss escalated more rapidly, with greater variability (Figure 2 ). When separating the samples between bicuspid and tricuspid aortic valve types, it appeared that both valve types were on the same curve.

The rapid increase and greater variability in energy loss at $>5.5 \mathrm{~cm}$ in aortic size resulted in a group of aneurysmal aortas with a wide range of energy loss indistinguishable by aortic size. Their histologic features were examined, and advanced medial degeneration was found in the aortas with elevated energy loss, with normal aortic wall architecture in the aortas with lower energy loss but the same aortic size (Figure 3).

The relationship between energy loss and aortic size remained once the size had been indexed to the body surface area (Figure 4). Indexing aortic size accounted for the concept that a $6-\mathrm{cm}$ aorta is not the same in a large individual as the same size aorta in a very small individual. Indexing resulted in a stronger, more linear, relationship with energy loss $\left(P<.0001, r^{2}=.78\right)$. One could still appreciate a clear increase in energy loss beyond an indexed aortic size of $3.25 \mathrm{~cm} / \mathrm{m}^{2}$. Despite indexing the aortic size, 1 sample was clearly far from the trend line. Histologic examination revealed extremely disorganized medial degeneration in this aorta (Figure 4, A). Another aorta of approximately the same age and indexed aortic size demonstrated uncompromised histologic features, with orderly elastin sheets and preserved smooth muscle cells.

Next, we quantified the changes in the underlying histologic features among a subset of the aneurysmal aortas by colorimetrically separating the components of the aortic wall into elastin, collagen, smooth muscle cells, and mucopolysaccharides. Individual components and the elastin/ collagen ratio were compared with the aortic size, energy loss, and modulus of elasticity. Increasing energy loss and increasing aortic size correlated significantly with increasing proportions of collagen (energy loss, $P=.004$, $r^{2}=.33$; size, $\left.P=.009, r^{2}=.27\right)$ and decreasing proportions of elastin (energy loss, $P=.003, r^{2}=.34$; size, $\left.P=.009, r^{2}=.27\right)$. The best correlations were found with increasing the collagen/elastin ratio (energy loss, $P=.0002, r^{2}=.49$; size, $P=.0007, r^{2}=.42$ ). No statistically significant relationship was found between the modulus of elasticity and aortic wall composition. Also, the mucopolysaccharide content did not correlate with aortic size or either of the biomechanical parameters.

\section{DISCUSSION}

The exact mechanisms of nontraumatic aortic complications have not been well delineated. Generally, a larger aortic size has been associated with a greater risk of dissection and rupture. Thus, clinicians have used aortic size almost exclusively to gauge the need for prophylactic surgery. This has occurred, despite the great heterogeneity in growth rates and lack of clarity of the natural history of ascending aortic aneurysms. Ascending aortic dissection and rupture occur when the mechanical integrity of the aorta is overcome by stresses on the wall. Aortic size is not a material property and thus is an inadequate surrogate for 

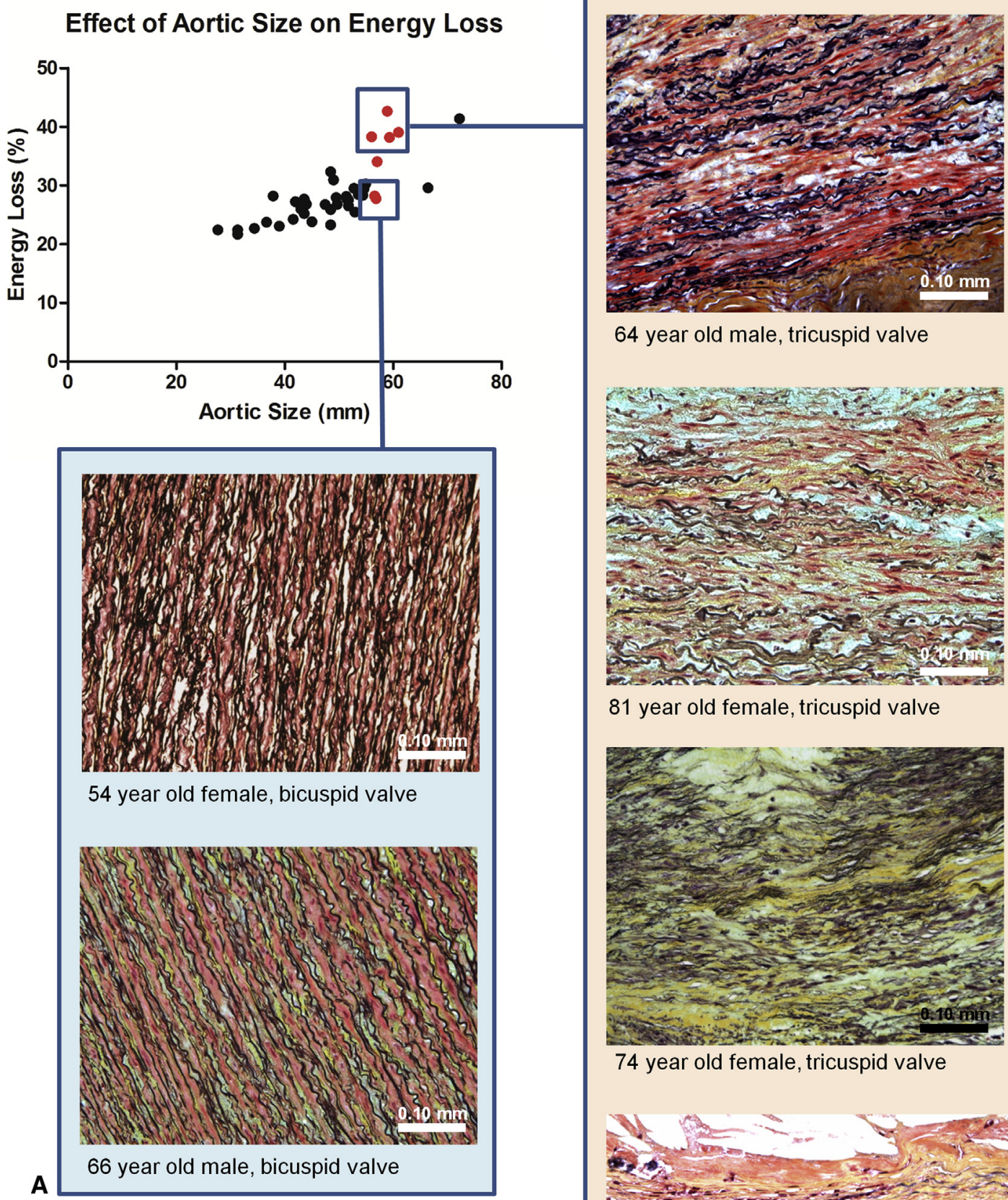

64 year old male, tricuspid valve

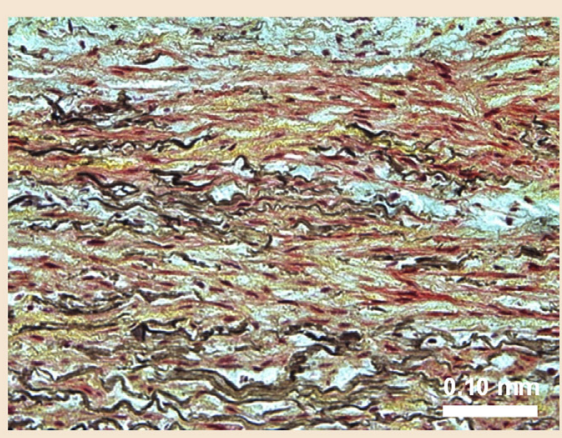

81 year old female, tricuspid valve

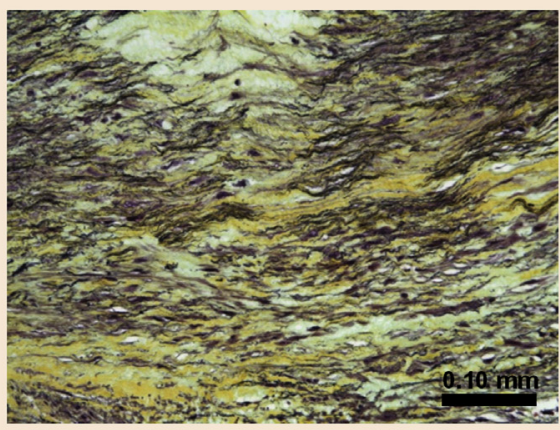

74 year old female, tricuspid valve

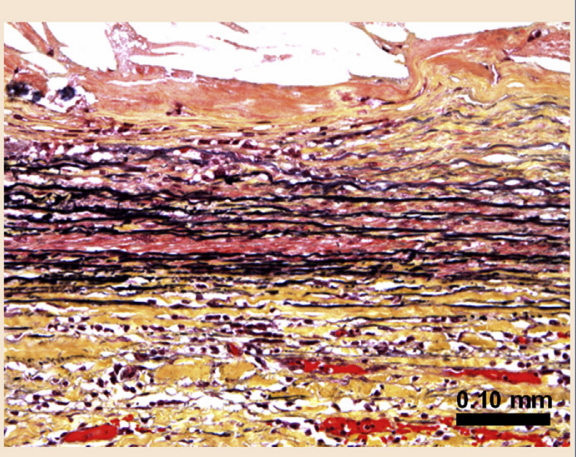

77 year old female, tricuspid valve

\section{B}

FIGURE 3. At $>5.5 \mathrm{~cm}$ in size, energy loss rapidly increased, with greater variability. The red dots represent aortas indistinguishable by aortic size but with a wide range in energy loss. Aortas with low energy loss, box (A), displayed normal histologic features with preserved elastic laminae (brown to black) and intact smooth muscle cells (red to purple). In contrast, aortas with elevated energy loss, box (B), display fragmentation of elastin, loss of smooth muscle cells, and replacement with collagen (yellow). 


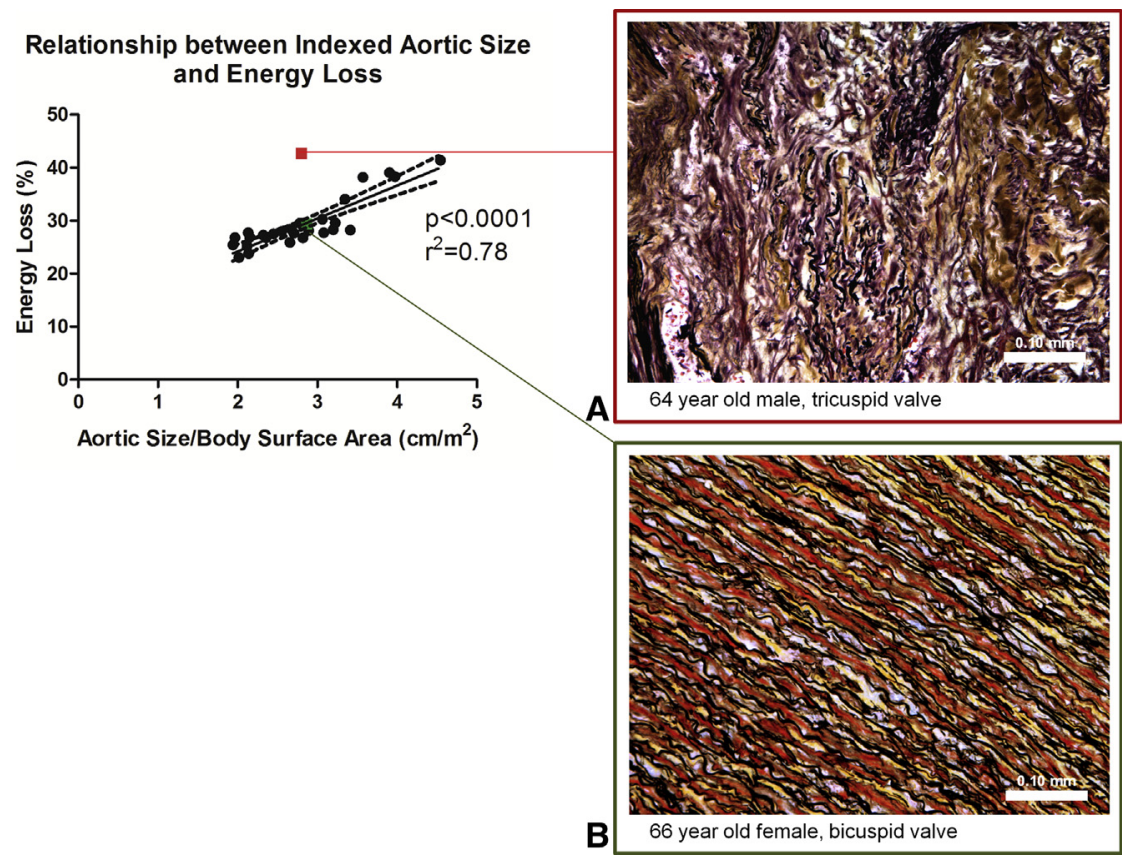

FIGURE 4. Once the aortic size has been indexed to the body surface area, the relationship between size and energy loss linearized, although an increase can still be appreciated after $3.25 \mathrm{~cm} / \mathrm{m}^{2}$. Energy loss pulled 1 sample off the trend line conspicuously as an outlier (red square). Box (A) shows the abnormal histologic features of the outlying sample, including fragmented elastin (black), disorganized smooth muscle cells (purple), and large amounts of disorganized collagen (yellow/brown). The green square represents a sample with a similar indexed aortic size, and box (B) shows the corresponding histologic features, with orderly elastic lamellae, orderly smooth muscle cells, and only moderate amounts of collagen.

wall stress. Therefore, biomechanics can provide mechanistic insight into aortic aneurysm complications and more sophisticated patient selection, reducing exposure to the real risks of even elective surgery.

Ex vivo biaxial tensile testing such as performed in the present study is the ideal experimental setting. The stress-strain characteristics of the tissue can be obtained under controlled loading or displacement over a wide range of amplitudes and frequencies without interference of surrounding tissue. Currently available medical imaging provides estimates of strain over the limited uncontrolled displacement of the cardiac cycle. Stresses are rough estimates determined from the blood pressure and rely on many assumptions of the vessel material properties. Although in vivo measures have shown good co-variance with patient age and aortic diameter, ${ }^{4-6,11,12}$ they have never been validated against the tissue material properties of the ascending aorta.

Aortic stiffness, characterized by an apparent modulus of elasticity, is an intuitive parameter thought to be one of the earliest markers of vessel disease, ${ }^{13}$ previously described in association with essential hypertension and aneurysms. ${ }^{8,14,15}$ However, how it is related mechanistically to the development of complications is unclear. Also, its nonlinear variation with strain presents significant difficulties in comparing it among studies and among patients and in providing meaningful in vivo measurements. In our study, the apparent modulus of elasticity demonstrated no correlation with the underlying histopathologic findings.

Energy loss is, by definition, a more stable robust variable less susceptible to noise. Potentially, its simplicity can make in vivo measurements more feasible and comparisons between studies and patients also easier. As a normalized measurement of hysteresis per cycle of loading and unloading, it represents the viscous component of the aortic wall. We found by analyzing the histologic findings that, unlike the apparent modulus of elasticity, energy loss did reflect the structural makeup of the aortic tissue, especially the collagen/elastin ratio. Poor efficiency in returning energy when cycled was associated with more advanced medial degeneration and greater collagen/elastin ratios. Whether it acts as a stimulus for vessel wall remodeling or is a byproduct of remodeling is unknown. However, if the amount of energy absorbed by the aortic wall exceeds the aorta's ability for repair, it would ultimately render the material more prone to failure.

The calculation of energy loss is completely independent of aortic size; however, a highly significant association was found between the 2 properties $(P<.0001)$. We demonstrated that at the high end of aortic size, aortas exhibited a rapid increase in the levels of energy loss. Additional examination showed that aortas with greater energy loss had much more medial degeneration than did their counterparts of the same size. 
By indexing the aortic size to the body surface area, we achieved greater linearization of the relationship between energy loss and aortic size $\left(r^{2}=.78\right)$. These results provide biomechanical support for the concept that the indexed size might be more important than the size itself. ${ }^{16}$ This is in contrast to the traditional parameter of apparent modulus of elasticity, which did not have any relationship with the indexed aortic size. The increase in energy loss seen at $3.25 \mathrm{~cm} / \mathrm{m}^{2}$, or its rapid increase beyond $5.5 \mathrm{~cm}$, might point to the mechanism behind the hinge-point in complication rates with increasing aortic size. ${ }^{17}$

An important finding was that energy loss was able to clearly separate an aortic sample with very pathologic changes seen on histologic examination. The energy loss was well off the curve expected, given either the size or indexed size, and the histologic changes were not seen in aortas of the same size. The severity of disease is unlikely to have been identified from the age, sex, and valve type of this 64-year-old male patient with a tricuspid valve. These results suggest that energy loss could provide additive value to examining the aortic size alone. On examination of Figure 2, one could hypothesize a 30\% level of energy loss would represent a cutoff other than size for recommending intervention. If such a cutoff was used, $18 \%$ of the aneurysmal aortas in our cohort would not have been removed. It is unknown what the clinical consequences of that would be at present. It is not certain whether and how the pathologic changes we observed would translate into aortic dissection. However, a contemporary case series of aortic dissection noted the importance of subadventitial collagen hyperplasia, offering some merit to the importance of an increased collagen/elastin ratio. ${ }^{18}$

Our results suggest that aneurysms associated with bicuspid aortic valves have energy loss similar to that of tricuspid valves at any given aortic size. Other studies of surgical specimens have found biomechanical differences between the 2 valve types in terms of stiffness, delamination strength, and tensile strength. ${ }^{19,20}$ We also found a difference in energy loss between bicuspid and tricuspid valves $(P=.02$, data not shown); however, when plotted against aortic size, the 2 valve types were on the same curve. Certainly, the incidence of aneurysms has been high among patients with bicuspid aortic valves, ${ }^{21}$ and compelling hemodynamic data exist on how wall stress differs in bicuspid aortopathy. ${ }^{22}$ However, whether a smaller aortic size is more significant in this population is debatable, ${ }^{23,24}$ as reflected by changes in the 2014 American Heart Association/American College of Cardiology valve guidelines, which raised the threshold for intervention for bicuspid aortopathy from $5.0 \mathrm{~cm}$ to 5.5 $\mathrm{cm} .{ }^{25}$ Aortopathy among patients with bicuspid aortic valves exhibits heterogeneity, ${ }^{26}$ and not all patients will merit early surgery. Energy loss might detect those who fall off the normal curve.
Our future objectives include establishing a noninvasive method of measuring metrics such as energy loss in vivo. We are currently validating both preoperative magnetic resonance imaging and intraoperative transesophageal echocardiographic data with our ex vivo data using a previously described method. ${ }^{5,27}$ Also, the genetic and biochemical factors that contribute to elevated energy loss need additional exploration. We envision refining risk stratification and surgical selection of candidates for ascending aortic aneurysm repair through the addition of simple biomechanical parameters to aortic size.

\section{CONCLUSIONS}

A highly significant association was found between energy loss and aortic diameter, with aortas exhibiting a rapid increase in energy loss levels at the high end of aortic size. Aortas with greater energy loss had much more medial degeneration than did their counterparts of the same size. Energy loss had a significant association with the underlying histologic features of the aorta. By demonstrating a measurable link between aortic function and structure, energy loss can provide an additional tool in distinguishing aortas at risk of complications.

\section{References}

1. Hiratzka LF, Bakris GL, Beckman JA, Bersin RM, Carr VF, Casey DE Jr, et al. 2010 ACCF/AHA/AATS/ACR/ASA/SCA/SCAI/SIR/STS/SVM guidelines for the diagnosis and management of patients with Thoracic Aortic Disease: a report of the American College of Cardiology Foundation/American Heart Association Task Force on Practice Guidelines, American Association for Thoracic Surgery, American College of Radiology, American Stroke Association, Society of Cardiovascular Anesthesiologists, Society for Cardiovascular Angiography and Interventions, Society of Interventional Radiology, Society of Thoracic Surgeons, and Society for Vascular Medicine. Circulation. 2010;121:e266-369.

2. Verma S, Yanagawa B, Kalra S, Ruel M, Peterson MD, Yamashita MH, et al. Knowledge, attitudes, and practice patterns in surgical management of bicuspid aortopathy: a survey of 100 cardiac surgeons. J Thorac Cardiovasc Surg. 2013; 146:1033-40.e4

3. Pape LA, Tsai TT, Isselbacher EM, Oh JK, O'Gara PT, Evangelista A, et al. Aortic diameter $>$ or $=5.5 \mathrm{~cm}$ is not a good predictor of type A aortic dissection: observations from the International Registry of Acute Aortic Dissection (IRAD). Circulation. 2007;116:1120-7.

4. Koullias G, Modak R, Tranquilli M, Korkolis DP, Barash P, Elefteriades JA. Mechanical deterioration underlies malignant behavior of aneurysmal human ascending aorta. J Thorac Cardiovasc Surg. 2005;130:677-83.

5. Oishi Y, Miyoshi H, Mizuguchi Y, Iuchi A, Nagase N, Oki T. Aortic stiffness is strikingly increased with age $>/=50$ years in clinically normal individuals and preclinical patients with cardiovascular risk factors: assessment by the new technique of 2D strain echocardiography. J Cardiol. 2011;57:354-9.

6. Petrini J, Jenner J, Rickenlund A, Eriksson P, Franco-Cereceda A, Caidahl K, et al. Elastic properties of the descending aorta in patients with a bicuspid or tricuspid aortic valve and aortic valvular disease. J Am Soc Echocardiogr. 2014;27:393-404.

7. Choudhury N, Bouchot O, Rouleau L, Tremblay D, Cartier R, Butany J, et al. Local mechanical and structural properties of healthy and diseased human ascending aorta tissue. Cardiovasc Pathol. 2009;18:83-91.

8. Khanafer K, Duprey A, Zainal M, Schlicht M, Williams D, Berguer R. Determination of the elastic modulus of ascending thoracic aortic aneurysm at different ranges of pressure using uniaxial tensile testing. J Thorac Cardiovasc Surg. 2011;142:682-6.

9. Martin C, Sun W, Pham T, Elefteriades J. Predictive biomechanical analysis of ascending aortic aneurysm rupture potential. Acta Biomater. 2013;9:9392-400. 
10. Tremblay D, Cartier R, Mongrain R, Leask RL. Regional dependency of the vascular smooth muscle cell contribution to the mechanical properties of the pig ascending aortic tissue. J Biomech. 2010;43:2448-51.

11. Oishi Y, Miyoshi H, Iuchi A, Nagase N, Ara N, Oki T. Negative impact of cardiovascular risk factors on left atrial and left ventricular function related to aortic stiffness-new application of 2-dimensional speckle-tracking echocardiography. Circ J. 2013;77:1490-8.

12. Petrini J, Yousry M, Rickenlund A, Liska J, Hamsten A, Eriksson P, et al. The feasibility of velocity vector imaging by transesophageal echocardiography for assessment of elastic properties of the descending aorta in aortic valve disease. J Am Soc Echocardiogr. 2010;23:985-92.

13. Cavalcante JL, Lima JA, Redheuil A, Al-Mallah MH. Aortic stiffness: current understanding and future directions. J Am Coll Cardiol. 2011;57: 1511-22.

14. Isnard RN, Pannier BM, Laurent S, London GM, Diebold B, Safar ME. Pulsatile diameter and elastic modulus of the aortic arch in essential hypertension: a noninvasive study. J Am Coll Cardiol. 1989;13:399-405.

15. Vorp DA, Schiro BJ, Ehrlich MP, Juvonen TS, Ergin MA, Griffith BP. Effect of aneurysm on the tensile strength and biomechanical behavior of the ascending thoracic aorta. Ann Thorac Surg. 2003;75:1210-4.

16. Davies RR, Gallo A, Coady MA, Tellides G, Botta DM, Burke B, et al. Novel measurement of relative aortic size predicts rupture of thoracic aortic aneurysms. Ann Thorac Surg. 2006;81:169-77.

17. Elefteriades JA. Natural history of thoracic aortic aneurysms: indications for surgery, and surgical versus nonsurgical risks. Ann Thorac Surg. 2002;74:S1877-80; discussion S92-8.

18. Schmitto JD, Popov AF, Coskun KO, Friedrich M, Sossalla S, Didilis V, et al. Morphological investigations of type A aortic dissection. Ann Thorac Cardiovasc Surg. 2010;16:331-4

19. Pasta S, Phillippi JA, Gleason TG, Vorp DA. Effect of aneurysm on the mechanical dissection properties of the human ascending thoracic aorta. J Thorac Cardiovasc Surg. 2012;143:460-7.

20. Pichamuthu JE, Phillippi JA, Cleary DA, Chew DW, Hempel J, Vorp DA, et al. Differential tensile strength and collagen composition in ascending aortic aneurysms by aortic valve phenotype. Ann Thorac Surg. 2013;96:2147-54.

21. Siu SC, Silversides CK. Bicuspid aortic valve disease. J Am Coll Cardiol. 2010; 55:2789-800.

22. Pasta S, Rinaudo A, Luca A, Pilato M, Scardulla C, Gleason TG, et al. Difference in hemodynamic and wall stress of ascending thoracic aortic aneurysms with bicuspid and tricuspid aortic valve. J Biomech. 2013;46: 1729-38.

23. Tzemos N, Therrien J, Yip J, Thanassoulis G, Tremblay S, Jamorski MT, et al Outcomes in adults with bicuspid aortic valves. JAMA. 2008;300:1317-25.

24. Michelena HI, Desjardins VA, Avierinos JF, Russo A, Nkomo VT, Sundt TM, et al. Natural history of asymptomatic patients with normally functioning or minimally dysfunctional bicuspid aortic valve in the community. Circulation. 2008; 117:2776-84

25. Nishimura RA, Otto CM, Bonow RO, Carabello BA, Erwin JP III, Guyton RA, et al. 2014 AHA/ACC guideline for the management of patients with valvular heart disease: a report of the American College of Cardiology/American Heart Association Task Force on Practice Guidelines. J Am Coll Cardiol. 2014;63: e57-185.

26. Fedak PW. Bicuspid aortic valve syndrome: heterogeneous but predictable? Eur Heart J. 2008;29:432-3.

27. Fattori R, Bacchi Reggiani L, Pepe G, Napoli G, Bna C, Celletti F, et al. Magnetic resonance imaging evaluation of aortic elastic properties as early expression of Marfan syndrome. J Cardiovasc Magn Reson. 2000;2:251-6.

\section{Discussion}

Dr Michael P. Fischbein (Stanford, Calif). Excellent presentation. I just have a couple questions.

So, an important finding of your study is that energy loss reflects the structural makeup of the aortic wall, specifically that the higher energy loss seen was associated with a higher collagen/elastin ratio. Also, some animal studies, specifically the abdominal aortic aneurysm model, have actually shown that increased collagen deposition was a protective response to decreased aneurysm growth.

And so I was wondering if you can comment or hypothesize whether this increase in collagen that you are seeing, is that a compensatory protective mechanism in the specimens or is this a key initiating event during aneurysm formation?

Dr Chung. Thank you for the question. I think that there is some compensatory mechanism. So, the starting insult, whether it is hypertension or an aneurysm and the patient has elevated wall stress, for some reason, we know that the aorta chooses to remodel or change. From what we understand, increased collagen deposition occurs, with destruction of smooth muscle cells and elastin fragmentation and mucopolysaccharide deposition.

So, this is probably a compensatory mechanism, but it has so far been not shown why this would be biomechanically a poor development.

I think that is what our study was able to show. It provides a mechanistic reason for why these changes should be a disadvantage. After all, collagen has greater tensile force. Why is it not more advantageous? I think we were able to show why or at least bring in some theory as to why.

Dr Fischbein. Then, for my second question, you mentioned a little bit at the end, talking about in vivo studies that you can do, transesophageal echocardiography or magnetic resonance imaging. I just wondered if you could just elaborate a little bit more about how you predict we will be able to use this in an in vivo, noninvasive method for patients.

Let us say if we had a patient with a bicuspid valve and perhaps a 4-cm aneurysm, how would you predict this will help us decide whether to resect that aneurysm?

Dr Chung. I think the key is to reproduce the stress-strain relationship using the in vivo imaging findings, and the stress part will require some invasive blood pressure monitoring.

So the ideal method is not known. We are using some speckle tracking with transesophageal echocardiography intraoperatively on our patients, and they also undergo preoperative magnetic resonance imaging now. So, hopefully, we will have that data for you soon.

However, the idea would be, obviously, to preoperatively obtain the images with blood pressure monitoring at the same time, to reproduce that stress-strain curve, and generate a very simple number, which might help you decide during operative planning.

Dr Todd K. Rosengart (Houston, Tex). Following up on Dr Fischbein's comments, you have shown clearly that energy loss correlates with the histologic features. Can you share with us from your knowledge of the published data why we would be so concerned about the histologic features in reference to the risk of rupture? That is not an area that you have discussed up until now.

Dr Chung. Why the histologic findings are important?

Dr Rosengart. Well, from your knowledge of the published data, how do those changes in histologic features correlate with the risk of rupture, which I think is the ultimate clinical goal here.

Dr Chung. I think that is just based on, I guess, our understanding of how aortic aneurysms develop dissection and rupture. I do not think you can say specifically that this equals rupture or dissection. I think we can just show that a relationship exists between the structure and function. 
You are right in saying that that does not mean necessarily that intervening on these aortas might prevent these complications. Thank you.

Dr Rosengart. Thank you.

Dr David J. Sugarbaker (Houston, Tex). I just have a brief question. Very nice presentation.

In terms of your energy loss, how would this correlate with a simple Law of Laplace calculation in terms of the diameter of the aorta in terms of your energy strain calculations?

Dr Chung. Right. So all Laplace's Law states is that the greater the diameter, the greater the wall tension, and that is where it ends really. It does not go any further and does not describe anything about the aortic wall itself.

Dr Sugarbaker. Right. But what I am wondering is, if you take your calculations and do a simple Law of Laplace calculation, is the increasing diameter of the aorta calculated by Laplace? Does that correlate with the energy loss that you have studied? In other words, can you correlate a simple Law of Laplace calculation to your findings regarding energy loss and your energy strain calculation?

Dr Chung. I am not really sure I understand that question.

Dr Sugarbaker. Do you see what I mean?

Dr Chung. So, you are saying?
Dr Sugarbaker. I am saying once you have the standard, once you know what the energy loss is from your energy strain calculations, can you correlate that to a simple Law of Laplace or to a simple increase in the diameter of the aorta?

Dr Chung. So, we are demonstrating it in relationship to the size of the aorta?

Dr Sugarbaker. Right.

Dr Chung. Right. So, all Laplace's Law shows is that larger aortas will have higher wall tension.

Dr Sugarbaker. Increasing wall tension.

Dr Chung. That is right.

Dr Sugarbaker. And does that wall tension correlate with your other findings?

Dr Chung. Yes. So, if you have increased-so-

Dr Sugarbaker. Do you see what I am getting at?

Dr Thomas K. Waddell (Toronto, Ontario, Canada). We do, but, Dr Sugarbaker, I am under strict instructions from the Society to keep the discussion on track.

Dr Sugarbaker. What do you want me to do, pull rank here or what?

Dr Waddell. No, that is okay. I am afraid we will not have time for any more questions. We just need to move ahead to the next talk. 